\title{
Features of the organization of bread wheat 5BS chromosome region carrying the leaf rust resistance gene Lr52
}

\author{
Maria Bragina \\ Kurchatov Genomics Center, \\ ICG SB RAS, Novosibirsk, Russia \\ koltunova@bionet.nsc.ru
}

\author{
Dmitriy Afonnikov \\ Kurchatov Genomics Center, \\ ICG SB RAS, Novosibirsk, Russia \\ NSU, Novosibirsk, Russia \\ ada@bionet.nsc.ru
}

\author{
Elena Salina \\ Kurchatov Genomics Center, \\ ICG SB RAS, Novosibirsk, Russia \\ salina@bionet.nsc.ru
}

\begin{abstract}
Despite the reference wheat genome sequencing and annotation, the search and chromosome localization of economically valuable traits loci in the wheat genome remains relevant. We reported the sequence structure of the $26.6-\mathrm{Mb}$ wheat chromosome $5 \mathrm{BS}$ subtelomeric region associated to leaf rust resistant genes through targeted sequencing. We show that this chromosomal region of leaf rust resistance and susceptible wheat lines were efficiently captured with sufficient coverage for the annotation of the sequenced region.
\end{abstract}

Keywords - bread wheat genome; chromosome 5BS; Lr52 gene; targeted sequencing

\section{Introduction}

The wheat reference cultivar 'Chinese Spring' genome published by the International Wheat Genome Sequencing Consortium (IWGSC, 2018) and SNP markers associated with economically valuable genes in wheat varieties and lines will contribute to solving important problems in genetics and breeding. To date, more than 30 genes that control a number of morphological and quantitative traits, resistance to abiotic and biotic environmental factors have been mapped on wheat chromosome 5B. Of particular interest is the distal region of its short arm (5BS) which bears a number of agronomically important genes, including genes conferring resistance to fungal diseases ( $\mathrm{Lr} 52, \mathrm{LrK} 1, \mathrm{Yr} 47$, Snn3, Pm30, H31, QTL for stem rust, rhizoctonia net blotch and loose smut of wheat). The mapping of DNA markers linked to the resistance genes on the 5BS reference pseudomolecule (RefSeq v1.0) allows the region where these genes reside to be identified. Our study was aimed to approbate targeted sequencing for the structure analysis of chromosome 5BS region with a length of about 26.6 Mb using lines with and without the Lr52 gene.

\section{Materials and methods}

Mapping population F4 (lines from the cross $\operatorname{LrW}(52) \times$ hybrid 215) was developed for chosen plants which are differ to resistant genes against fungal diseases located on target region of 5BS. We selected 5 plants with $\operatorname{Lr} 52$ and 5 plants without Lr52 according to data of KASP and SSR genotyping together with screening for resistance (benzimidazole $(0.035$ $\% \mathrm{w} / \mathrm{v})$ ). Genomic DNA was isolated from the young leaves of individual plants using the Kleargene plant 96-well plate DNA extraction kit (LGC Group), following the manufacturer's protocol.

Target sequencing was performed using the SeqCap EZ Target Enrichment System (Roche) to enrich a $26 \mathrm{Mb}$ of $5 \mathrm{~B}$ chromosome subtelomeric region. The Roche company developed probes to cover the region under study on the basis of the reference genome. As a result, the maximum size of covered regions was about $8 \mathrm{Mb}$, mainly due to repeats. The 150 -bp pairedend sequencing of the obtained library was performed on an Illumina NextSeq 550 platform (ICG SBRAS).

\section{Results and Discussion}

The wheat lines of population F4 (lines from the cross $\operatorname{LrW}(52) \times$ hybrid 215 ) were captured with probes developed by Roche to cover the region under study on the basis of the reference genome. As a result of sequencing, an average of $4.28 \mathrm{~Gb}$ and $3.51 \mathrm{~Gb}$ of reads was obtained for the wheat lines with Lr52 and without Lr52, respectively. The total size of data obtained was about $39 \mathrm{~Gb}$. The proportions of sequences with $\mathrm{Q}>30$ were 78.95 and $78.52 \%$, and the mean quality score Q values were 31.44 and 31.35 for the wheat lines with Lr52 and samples without Lr52, respectively. After preprocessing and quality control using the FASTXtoolkit utility, the reads obtained were mapped onto the studied region of the wheat reference genome using the BWA-MEM program (Li and Durbin, 2009). On average, $70 \%$ of the reads were not mapped, $7-9 \%$ were mapped one time and $20 \%$ more than once for samples with and without Lr52. In the target regions, 10 wheat plants had narrow mean coverage ranges, $40 \times$ and $48 \times$, for the leaf rust resistant and susceptible samples, respectively.

To visualize the mapping of reads to the reference genome, the studied region was divided into 100 bases long segments, each of which was assigned a different color depending on the sequence matching (Fig 1).

Fig. 1. Study of the gene $\operatorname{Lr} 52$ localization region.

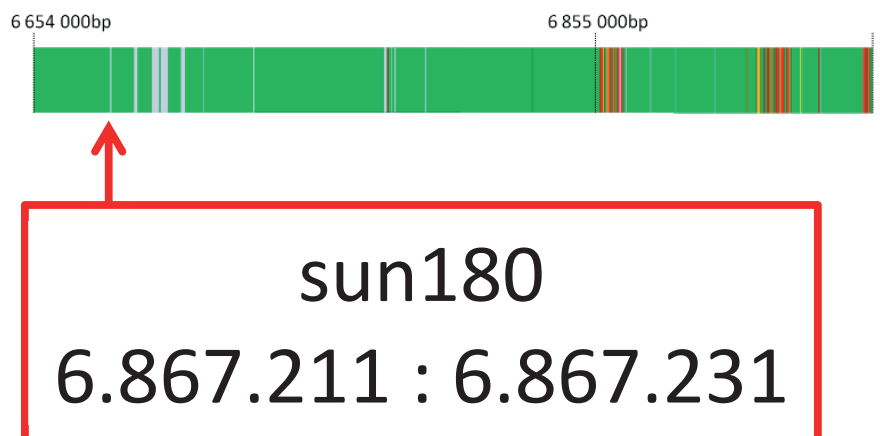

Fig. 2. Example of the reads mapping to the wheat reference genome on a plot with coordinates $6654000 \mathrm{bp}-6956436 \mathrm{bp}$. Green areas indicate the coincidence of the reference genome sequences, plants with Lr52 and 5 plants without Lr52; red color means the coincidence of the reference genome sequences and rust-susceptible plants without Lr52 gene and the absence of mapped sequences in this region in resistant samples; white - the gaps of a reference sequence in this region. Also the sun180 marker, which co-segregates with the Lr52 gene is indicated.

The $26.6 \mathrm{Mb}$ region was analyzed in NCBI, Ensembl Plants, GrainGenes, UniProt, PlantPAN 3.0, PlantPRe, PRGdb, psRNAtarget, miRBase, PmiRExAt databases. In result about 20.000 SNP were found to be dispersed in the 5BS distal region About 500 candidates for major resistance genes 
with the domains NBS, CC, LRR, Tm and kinase domains were revealed and about 30.000 potential exones, 5.500 putative genes and 400 miRNA were found. In the $6.6 \mathrm{Mb}-$ $7 \mathrm{Mb}$ region several microRNAs were found only in samples resistant to leaf rust, the sequences of which differed from the sequences of the reference genome and samples without Lr52 gene by SNP that are located in the miRNA stem-loop. Next, an automatic annotation was carried out using the FGENESH 2.6 program, which revealed differences between the samples in the area with $6.85 \mathrm{Mb}-6.86 \mathrm{Mb}$ coordinates. For samples without $\operatorname{Lr} 52$ gene, a potential gene is located in this segment and there is no coincidence in databases. In addition, samples with $\operatorname{Lr} 52$ gene contained a deletion in the $6.91 \mathrm{Mb}-6.93 \mathrm{Mb}$ region in which samples without Lr52 gene had annotated TRAESCS5B02G005400 gene that encodes a protein including two putative kinase domains. This gene is homologous to wheat stem rust resistant gene Sr60 from
Triticum monococcum and Sr60 has a deletion including the last two exons in Chinese Spring. So we may propose some versions of leaf-rust resistance mechanism.

\section{Acknowledgment}

Supported by the RFBR (17-04-00507).

\section{References}

[1] Li H., Durbin R. Fast and accurate short read alignment with Burrows Wheeler transform. Bioinformatics. 2009;25(14):1754-1760.

[2] The International Wheat Genome Sequencing Consortium. Shifting the limits in wheat research and breeding using a fully annotated reference genome. Sci. 2018;361(6403). 